\title{
Surface plasmon resonance-based DNA microarrays: Comparison of thiol and phosphorothioate modified oligonucleotides
}

\author{
K. L. Jiménez-Monroy ${ }^{*, 1,2, \dagger}$, A. Kick ${ }^{*, 1,3, \dagger}$, K. Eersels ${ }^{2}$, B. van Grinsven², P. Wagner², and M. Mertig ${ }^{1,2}$ \\ ${ }^{1}$ Professur für Physikalische Chemie, Mess- und Sensortechnik, Technische Universität Dresden, 01062 Dresden, Germany \\ ${ }^{2}$ Hasselt University, IMO-IMOMEC, Wetenschapspark 1, 3590 Hasselt, Belgium \\ ${ }^{3}$ Kurt-Schwabe-Institut für Mess- und Sensortechnik e.V., Kurt Schwabe- Strasse 4, 04736 Waldheim, Germany
}

Received 11 December 2012, revised 20 March 2013, accepted 23 March 2013

Published online 3 May 2013

Keywords DNA chip, DNA hybridization, microarray, phosphorothioate, surface plasmon resonance, thiol

* Corresponding author: e-mail kathia.jimenezmonroy@uhasselt.be, Phone: +32 112688 43, Fax: +32 11268899

**e-mail kick@ksi-meinsberg.de, Phone: +49-34327-608 173, Fax: +49 34327608131

${ }^{\dagger}$ Both authors contributed equally to this work.

We present the use of thiol-modified and phosphorothioate (PT)-modified oligonucleotides for building DNA microarrays on the gold surface of surface plasmon resonance (SPR) chips. PT-modified oligonucleotides (PTOs) have several advantages in comparison to thiol-modified ones. They do not form disulfides and the PT groups can be introduced in any desired position of the molecular backbone of the oligonucleotide. Additionally, modifications with PT groups are not as costintensive as thiol groups. The direct immobilization of the oligonucleotides via thiol and PT groups is compared. The affinity of the PT groups to gold is lower than that of thiol groups. Nevertheless, the hybridization kinetics of a model polymerase chain reaction (PCR) product could be studied in real time on a DNA microarray for both types of modified oligonucleotides. This contributes to clarification of previous, contradictory reports on the use of PTOs for their attachment to gold surfaces. The immobilization of PTOs could be improved by the introduction of iodoacetylated surfaces, which are reactive to bind PTs as well as thiols with high efficiency. Furthermore, the influence of the probe structure on the probe density and the hybridization was investigated.
1 Introduction Rapid and label-free detection of DNA hybridization can be a valuable tool for medical diagnostics and DNA interaction research in general. Realtime detection of DNA hybridization on multiple spots in parallel is achieved by using surface plasmon resonance (SPR) biosensors based on DNA microarrays. SPR sensors are refractometric sensing devices, requiring the functionalization of a metal film sensor surface, usually gold. These types of sensors measure changes of refractive index at this sensor surface with a very high sensitivity. SPR biosensors can be found in diverse fields of application in medical diagnostics, environmental monitoring and food safety. SPR is one of the main optical biosensor technologies [1].

The optical phenomenon of SPR occurs on noble metal surfaces. When light strikes the surface of a certain metal, there is a minimum in the angular intensity spectrum of the reflected light. The angle of this minimum (resonance angle) is a property of the plasmon resonance and depends on the refractive index of the medium and the evanescent wavelength used (about $200 \mathrm{~nm}$ ). The adsorption of biomolecules to this interface results in a change of the refractive index, which can be detected as a shift of the resonance angle.

Gold is the most widely used metal for the coating of SPR sensors because of its chemical stability against oxidation. Mostly, the attachment of organic molecules to gold is achieved via thiol groups. We report on an approach to create a DNA microarray for the detection of DNA hybridization based on SPR. Thiol-modified oligodeoxynucleotides (ODNs) are often used for the detection of DNA oligonucleotides on different transducer platforms based on impedance spectroscopy [2], voltametric detection [3, 4], SPR [5], or microgravimetric detection [6]. 
Thiol-modified ODNs are susceptible to enzymatic degradation, oxidation, or formation of disulfides. The disulfides can still bind to gold but they are not reactive nucleophiles and cannot be used for further modifications with electrophilic groups. Thiol groups are usually introduced to $3^{\prime}$ - or $5^{\prime}$-ends of ODNs. It is also possible to introduce them internally but this is more costly $[7,8]$.

The use of phosphorothioate (PT) modified ODNs (PTOs) can be beneficial in comparison to using thiolmodified ODNs. PTs withstand enzymatic degradation by endonucleases and do not oxidize [9]. They do not form disulfides, and are reactive nucleophiles. Furthermore, PT groups can be introduced to variable positions in the backbone during the synthesis of PTOs, and this is much cheaper compared to the introduction of thiol groups $[9,10]$. PT groups can be used as nucleophiles for immobilization of PTOs on, e.g., bromoacetylated surfaces. It is possible to use this chemistry to build microarrays for enzyme-mediated reactions on DNA chips [11]. Hairpin structured probes showed higher efficiency during enzymatic reactions than linear probes. The PT groups were introduced into the loop region of those hairpins to enable the covalent immobilization to a bromoacetylated surface. The PTO part which was responsible for the hybridization to target DNA should stay single-stranded and extend from the sensor surface $[12,13]$. Different approaches are described in literature to immobilize PTOs on gold. The most straightforward method is based on the interaction of gold with the sulfur of PT groups. Direct binding of PTOs to gold nanoparticles is described in literature [14-17]. Furthermore, PTOs were directly immobilized on flat gold surfaces $[18,19]$.

However, these findings do not fully correspond to Lee et al. [20], which reported that the distance of gold nanoparticles on DNA could not be controlled and a low coverage of the gold nanoparticles was detected when only PTOs were used. Therefore, there were efforts to improve the functionalization of gold nanoparticles to increase the yield of the bound PTOs. This was achieved by using a bifunctional linker containing one thiol and one iodoacetyl moiety to bind covalently to PT groups.

In order to clarify these contradictions, we compare the direct immobilization of PTOs and thiol-modified ODNs on the flat gold surface of an SPR chip. Furthermore, we developed an iodoacetyl surface for a more efficient immobilization of PTOs. The hybridization of a polymerase chain reaction (PCR) product was monitored by real-time SPR measurements proving that functionalization of the gold surface was successful. This model PCR product is 300 base pairs (bp) long and contains an additional 5 '-single stranded overhang with a tag sequence. This tag sequence is complementary to an antitag sequence of the immobilized DNA probes of the microarray (Fig. 1).

In order to assess the efficiency of the hybridization of complementary tags with the different antitags under study, the kinetics of the different SPR signals were analyzed. Nilsson et al. analyzed DNA hybridization kinetics by means of SPR and demonstrated that hybridization occurs more

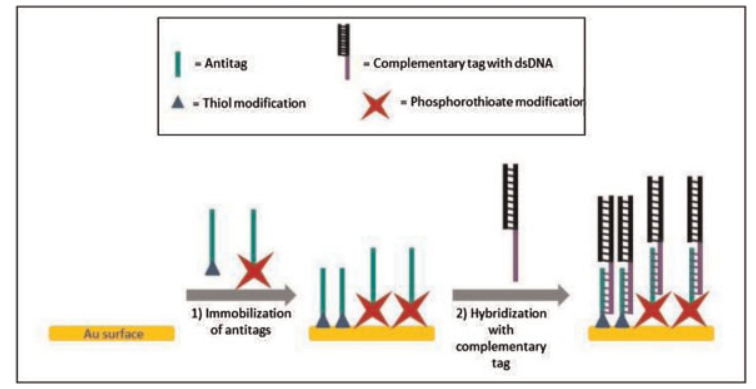

Figure 1 Schematical illustration of the immobilization of modified (SH or PT) antitags to the gold surface of a SPR chip and their hybridization with complementary tags.

efficiently toward the $3^{\prime}$-end of the probe DNA [21]. More recently van Grinsven et al. used denaturation kinetics to study DNA duplex stability and concluded that denaturation occurs faster in non-complementary DNA sequences [22].

\section{Experimental procedure}

\subsection{Materials and methods}

2.1.1 Synthesis of the PCR product $300 \mathrm{bp}-1$ long PCR products, containing a protruding tag ( $5^{\prime}$-ATAGGCTCTCCAGAATAAGGTCTCG-3') were synthesized by asymmetric PCR, using one primer pair (biomers.net) and the pUC19 vector (Invitrogen). One of the primers was modified at its $5^{\prime}$-position with the tag sequence separated by a hexaethylene glycol (HEG) spacer: 5'-tag-HEG-AGCCTGAATGGCGAATGG- $3^{\prime}$. The sequence of the other primer was 5'-GACATTAACCTATAAAACTAGGCGTATCA$3^{\prime}$. The PCR product was purified by using a commercial kit (Qiagen) and was quantified by UV absorbance (BioPhotometer, Eppendorf).

\subsubsection{Immobilization of the oligonucleotides} The immobilization solutions in deionized water contained $50 \mu \mathrm{M}$ oligonucleotides, $100 \mathrm{mM} \mathrm{MgCl}_{2}$ and $5 \%$ glycerol. These solutions were deposited on the modified or bare gold surface of an SPR chip by using the nanoliter dispenser NanoPlotter 2.1 (GeSiM mbH, Germany). The SPR spectrometer averages over the length of $1 \mathrm{~mm}$ long line shaped spots. Thus, lines of the immobilization solutions could be generated by depositing $50 \mathrm{pL}$ droplets with a pitch of $36 \mu \mathrm{m}$ (to enable an overlap of the droplets) and a column pitch of $72 \mu \mathrm{m}$. The width of these lines varied depending on the wettability of the used surface. In order to avoid cross contamination of different oligonucleotide solutions on the SPR chip, a pitch of at least $200 \mu \mathrm{m}$ was chosen between the lines. Each antitag is immobilized in triplicate on the surface of the chip (three lines of 24 spots). The surface area of the sensitive gold layer of the chip is $36 \mathrm{~mm}^{2}[23-25]$.

After immobilization of antitags on bare gold chips a blocking step using mercaptohexanol $(1 \mathrm{mM})$ was performed for $30 \mathrm{~min}$ before hybridization experiments.

All hybridization measurements were performed at $25^{\circ} \mathrm{C}$, using $60 \mathrm{mM}$ of $\mathrm{MgCl}_{2}$ buffer solution, a tag 


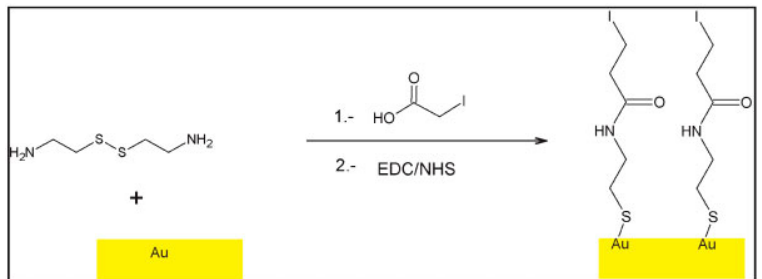

Figure 2 Chemical reaction on the gold layer to form a iodoacetyl brush type structure.

concentration of $2.5 \mathrm{ng} \mu \mathrm{L}^{-1}(13 \mathrm{nM})$ and PCR products of $300 \mathrm{bp}$.

2.2 Chemical functionalization All gold surfaces were cleaned with ethanol and dried by using nitrogen gas. Moreover, two functionalization strategies were applied independently in order to prepare iodine reactive gold surfaces through self-assembled monolayers (SAMs).

\subsubsection{Brush type $=$ cystamine/NHS/EDC/iodoacetic} acid (CA/NHS/EDC/la) After cleaning, a $3 \mathrm{mM}$ cystamine solution was added to the gold surface. After $1 \mathrm{~h}$, the surface was washed with deionized water and dried. Next, the surface was covered with a solution containing $10 \mathrm{mM} \mathrm{NHS \text {, }}$ $10 \mathrm{mM}$ EDC, and $10 \mathrm{mM}$ Ia. The reaction was stopped after $1 \mathrm{~h}$ by washing with deionized water. The scheme of these steps are summarized in Fig. 2.

Succinic anhydride blocking solution. This solution was prepared as follows in the literature [26]. To $1.9 \mathrm{~mL}$ of $N$ methyl pyrrolidinone (NMP) was added $33.1 \mathrm{mg}$ of succinic anhydride and stirred for $10 \mathrm{~min}$. Then $0.1 \mathrm{~mL}$ of sodium borate $(0.02 \mathrm{M}, \mathrm{pH}=8)$ was added to the mixture. For the chips functionalized with cystamine, the blocking treatment lasted $30 \mathrm{~min}$ and afterwards the chips were rinsed with deionized water and blown dry with nitrogen.

2.2.2 Tripod type $=$ (3-iodopropyl)-trimethoxysilane A solution of $1 \mathrm{mM}$ mercaptoundecanol $(\mathrm{MuOH})$ was added to a clean gold surface. After $2 \mathrm{~h}$, the surface was washed with ethanol and dried. The next step was the addition of a solution containing 10\% silane in 95\% ethanol/

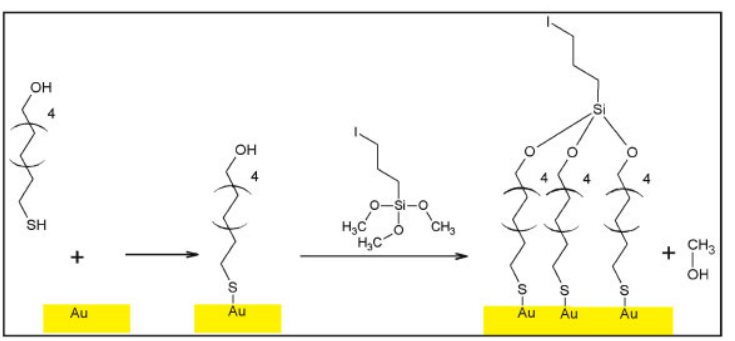

Figure 3 Two-step reaction to prepare an iodosilane-functionalized gold surface.

deionized water to the surface and incubation for $2 \mathrm{~h}$, followed by washing and drying of the sample. The steps mentioned above are summarized in Fig. 3. After functionalization the probes were immobilized on the functionalized gold surface. Before hybridization a blocking step was performed using mercaptohexanol $(1 \mathrm{mM})$ for $30 \mathrm{~min}$ in order to avoid unspecific absorption to the functionalized gold surface.

2.3 Antitags ("at") immobilization The antitags were designed taking into account three features: (i) "at" modification: either SH or PT was incorporated into the antitag. (ii) Spacers: consisting of eight nucleotide bases (A8 - adenine or T8 - thymine); (iii) structure modification: linear or hairpin (hp) structure. A sequence of nucleotides, different to the antitag of interest was used as a control "nt". The names and sequences of all used antitags are listed in Table 1.

The geometrical structure of at-hp12-5PT-0 is shown in Fig. 4.

2.4 SPR setup The SPR setup used for detecting hybridization of tags with "at" is described in Fig. 1. Gold surfaces were deposited on a TOPAS ${ }^{\odot}$ chip and functionalized in order to improve immobilization of "at" to the surface. PCR products containing tags were transported to the surface by means of a microfluidic system. Hybridization was detected as a change of the refractive index at the surface. In addition, reference measurements were performed by using SH- and PT-modified "at" immobilized directly to a gold surface. Hybridization with the $300 \mathrm{bp}$-long PCR product was analyzed by SPR and the signal intensity is given in refractive

Table 1 List of antitags used for hybridization experiments.

abbreviation

(at)

(nt)

at- ${ }^{\prime}{ }^{\prime} \mathrm{SH}$

nt- $3^{\prime} \mathrm{SH}$

at- $\mathrm{A} 8-3^{\prime} \mathrm{SH}$

at- $\mathrm{T} 8-3^{\prime} \mathrm{SH}$

at-A8-5PT

at-T8-5PT

at-hp12-5PT-0 sequence $5^{\prime}$ to $3^{\prime}$

\author{
ATA GGC TCT CCA GAA TAA GGT CTC G \\ TAG GTG GTA CGT CTA TTC CTG GTC CTT TTT TTT \\ (at) $-3^{\prime} \mathrm{SH}$ \\ (nt)-3'SH \\ (at)-AAA AAA AA- $3^{\prime} \mathrm{SH}$ \\ (at)-TTT TTT TT- $3^{\prime}$ SH \\ (at)-AAA * $\mathrm{A}^{*} \mathrm{~A}^{*} \mathrm{~A}{ }^{*} \mathrm{~A}{ }^{*} \mathrm{~A}$ \\ (at)-TTT ${ }^{*} \mathrm{~T}^{*} \mathrm{~T}^{*} \mathrm{~T}^{*} \mathrm{~T}^{*} \mathrm{~T}$ \\ (at)-GAT CGA TCG ATC ${ }^{*} \mathrm{G}^{*} \mathrm{~T}^{*} \mathrm{~A}{ }^{*} \mathrm{~A}^{*} \mathrm{GA}$ TCG ATC GAT C
}




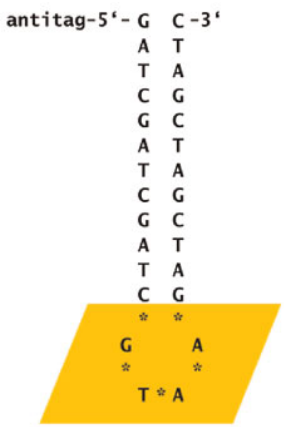

Figure 4 Representation of at-hp12-5PT-0 with a hairpin. The loop is formed by four bases $\left({ }^{*} \mathrm{G}^{*} \mathrm{~T}^{*} \mathrm{~A}^{*} \mathrm{~A}^{*}\right)$ with five PT groups.

index units (riu). The data received by the CCD camera of the SPR set-up are used to calculate the maximum SPR signal of every probe at a defined position [23-25].

\section{Results and discussion}

3.1 Calculation of antitag surface coverage The expected number of immobilized antitags per area was calculated based on the theoretical values of the so called Flory radius $\left(R_{\mathrm{f}}\right)$. It is a well-known fact that ssDNA has a lower persistence length $\left(l_{\mathrm{p}}\right)$ in comparison to dsDNA. The persistence length for ssDNA at a buffer concentration of $60 \mathrm{mM}$ is $2.04 \mathrm{~nm}$ [27]. The antitags used in this study all have less than 100 nucleotides, hence will appear in the coiled up state [28]. The Flory radius was calculated using the following formula: $R_{\mathrm{f}}=\left(l_{\mathrm{p}} \times L / 3\right)^{1 / 2}$ [29]. The $R_{\mathrm{f}}$ values and the calculated maximum coverage are summarized in Table 2 for each of the antitags used in the experiments.

The results summarized in Table 2 correspond well to the papers of van Grinsven et al. [29], Vermeeren et al. [30] and the references therein. The calculation is based on the assumption that DNA can only form a monolayer; however, results in literature show that experimental surface coverage data can exceed the theoretical maximum values given in Table 2 [29]. The drawback of this theoretical calculation is the fact that the hairpin structured antitag does not fully fit the model. The calculated maximal surface coverage values can therefore be regarded as an approximation.

To determine the actual experimental value of the antitag surface coverage, the SPR signal was analyzed upon
Table 2 Calculated Flory radius and calculated maximal antitag surface coverage.

\begin{tabular}{llll}
\hline probe & $N(\mathrm{bp})$ & $R_{\mathrm{f}}(\mathrm{nm})$ & $\begin{array}{l}\text { theoretical maximal } \\
\text { coverage }\left(\mathrm{at} \mathrm{mm}^{-2}\right)\end{array}$ \\
\hline (at)-3'SH & 25 & 2.4 & $5.5 \times 10^{10}$ \\
(nt)-3'SH & 25 & 2.4 & $5.5 \times 10^{10}$ \\
(at)-A8-3'SH & 33 & 2.7 & $4.4 \times 10^{10}$ \\
(at)-T8-3'SH & 33 & 2.7 & $4.4 \times 10^{10}$ \\
(at)-A8-5PT & 33 & 2.7 & $4.4 \times 10^{10}$ \\
(at)-T8-5PT & 33 & 2.7 & $4.4 \times 10^{10}$ \\
(at)-hp12-5PT-0 & 53 & 3.5 & $2.5 \times 10^{10}$ \\
\hline
\end{tabular}

immobilization of different types of antitags to bare gold, iodoacetyl-, and iodosilane-functionalized gold surfaces. The results are summarized in Table 3 .

The surface densities of probe DNA in Table 3 were calculated using the shift in response units upon adsorption of probe DNA on bare gold as well as iodoacetyl and iodosilane functionalized gold surfaces. Taking into account that the riu units are equivalent to: 1 riu $=0.01$ Response Unit (RU) and $1 \mathrm{RU} \sim 1 \mathrm{pg} \mathrm{mm}^{-2}$, then from the raw data, the angular shift is calculated, taking into account that the standard angular shift for gold on the TOPAS ${ }^{\odot}$ is $\sim 200 \mathrm{~nm}$. Comparing the values to the values calculated by using the Flory radius, only gives a low coverage as compared to the theoretical maximum. This is not surprising given the immobilization route of the antitags, covering only about 1/491th of surface area of the sensitive gold layer [25].

The values in Table 3 clearly show that the difference in surface coverage when using different probes corresponds well with the maximum values calculated by the Flory radius in Table 2. However, the PT-functionalized antitag employing a T8 linker shows a lower antitag surface coverage as compared to the same antitag with an A8 linker. This can be explained by the fact that adenine shows a higher affinity for gold then thymine. This does not seem to play a role for SH-functionalized antitag, probably because of differential orientation.

Furthermore, iodosilane functionalized gold surfaces display a higher antitag surface coverage in comparison to bare gold surfaces.

In addition, iodoacetyl functionalization of the gold SPR chip leads to an increased immobilization of antitags to the

Table 3 Experimental values for antitag surface coverage for different antitags on bare gold, iodosilane-, and iodoacetyl-functionalized SPR chips.

\begin{tabular}{|c|c|c|c|}
\hline probe & gold $\left(\right.$ at $\mathrm{mm}^{-2}$ ) & iodosilane $\left(\right.$ at $\mathrm{mm}^{-2}$ ) & iodoacetyl (at $\mathrm{mm}^{-2}$ ) \\
\hline (at) $-3^{\prime} \mathrm{SH}$ & $(4.4 \pm 0.1) \times 10^{7}$ & $(5.8 \pm 0.1) \times 10^{7}$ & $(9.9 \pm 0.1) \times 10^{7}$ \\
\hline (nt) $-3^{\prime} \mathrm{SH}$ & $(4.2 \pm 0.2) \times 10^{7}$ & $(5.3 \pm 0.3) \times 10^{7}$ & $(8.0 \pm 0.2) \times 10^{7}$ \\
\hline (at)-A8-3'SH & $(3.5 \pm 0.1) \times 10^{7}$ & $(4.6 \pm 0.2) \times 10^{7}$ & $(6.9 \pm 0.2) \times 10^{7}$ \\
\hline (at)-T8-3'SH & $(3.3 \pm 0.2) \times 10^{7}$ & $(4.9 \pm 0.2) \times 10^{7}$ & $(6.4 \pm 0.2) \times 10^{7}$ \\
\hline (at)-A8-5PT & $(2.7 \pm 0.2) \times 10^{7}$ & $(4.5 \pm 0.1) \times 10^{7}$ & $(7.4 \pm 0.1) \times 10^{7}$ \\
\hline (at)-T8-5PT & $(2.0 \pm 0.2) \times 10^{7}$ & $(4.6 \pm 0.1) \times 10^{7}$ & $(7.2 \pm 0.2) \times 10^{7}$ \\
\hline (at)-hp12-5PT-0 & $(1.8 \pm 0.1) \times 10^{7}$ & $(2.6 \pm 0.2) \times 10^{7}$ & $(4.9 \pm 0.2) \times 10^{7}$ \\
\hline
\end{tabular}


surface in comparison to iodosilane functionalized gold surfaces. This can be explained by the fact that iodosilane functionalization is a more bulky route; the tripod structure leads to a more spread distribution of immobilized antitags.

Finally, chemically functionalized gold SPR chips seem to be preferable surfaces for the immobilization of PTfunctionalized antitags, showing a comparable antitag surface coverage as compared to the coverage using $\mathrm{SH}$ functionalized antitags. These findings can be explained by the fact that chemical functionalization creates a preferable surface for PT-functionalized antitag immobilization.

3.2 Reference measurements As mentioned above, reference measurements on bare gold surfaces were performed. Hybridization of $300 \mathrm{bp}$ PCR products to various types of "at" (described in Table 1) was analyzed. It was found that hybridization occurred within a short time span. Summation of these hybridization related results are given in Fig. 5.

In order to have a clear insight into the difference in hybridization detection using different antitags, the maximum values of the SPR signal difference for every probe are calculated and summarized in Fig. 6. The data in this graph show a higher signal for linear ssDNA with SH-modifications than for antitags containing PT-modifications. The values in Table 3 clearly indicate that this is a result of a lower antitag surface coverage for PT-functionalized antitags. In addition, higher signals were observed on spots where PT-modified antitags with A8 spacers were immobilized, when compared to spots containing PT-modified antitags with T8 spacers, corresponding well to the values summarized in Table 3. The absence of this effect for SH-functionalized antitags lays in the fact that these antitags are not susceptible to a better affinity of the linker region to the gold surface, this is confirmed by the data in Table 3 .

The signal in Fig. 6 for the hp-structured antitags was lower than expected from a previously reported investigation on hp antitags, where it was stated that higher hybridization signals should be observed using hp structures in comparison

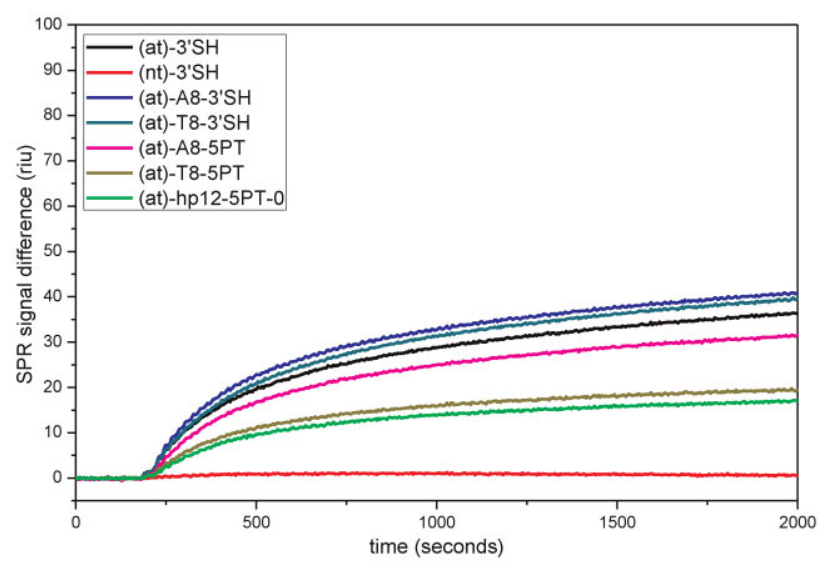

Figure 5 Hybridization kinetics (SPR signal in riu vs. time) for different antitags immobilized on top of a bare gold surface.

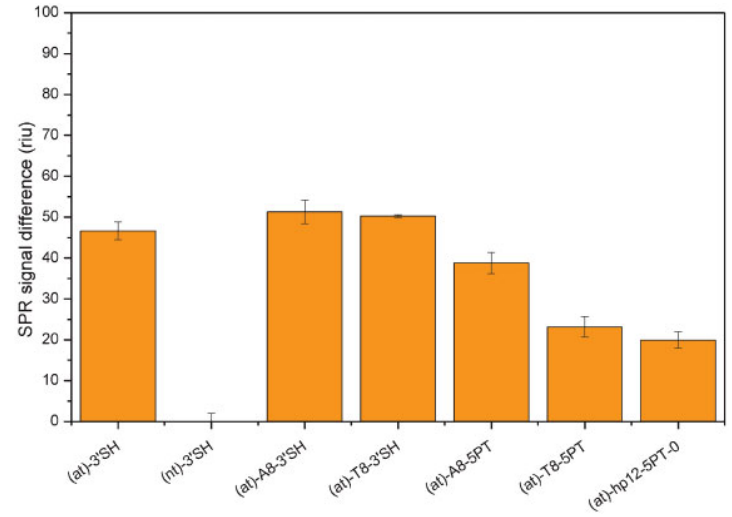

Figure 6 SPR signal difference (riu) after hybridization on spots, with the respective "at" immobilized on bare gold surface.

to linear antitags [31-33]. The use of five PT-groups as the anchoring structure might be a possible explanation for this observed discrepancy. This anchoring structure might lack the proper orientation when bound to the gold surface, causing more steric hindrance for the tag when hybridizing with the anti-tag sequence.

However, we presume that the main reason for the lower hybridization signal of the "at" with the spacer and stemloop structure is their Flory radius $\left(R_{\mathrm{f}}\right)$.

Finally, these results show that thiol-modified oligonucleotides as well as PTOs can be immobilized on the gold surface and that the hybridization detection is possible with both kinds of modification. On spots with PTOs, the hybridization signals are considerably lower. This indicates that the corresponding probe density is lower than that on spots with thiol-modified oligonucleotides as shown in Table 3. Thus, PT groups result in a less efficient immobilization of probe DNA. With respect to the reports of Lee et al. [20] and other researchers describing the interaction of PTOs with gold nanoparticles [14-17] the efficiency of this attachment could strongly depend on the incubation time, probe structure and concentration. Nevertheless, there are supporting reports which present the immobilization of PTOs on flat gold surfaces [18, 19].

In accordance with these publications, we could use PTOs directly on bare gold surfaces as probe DNA for hybridization detection. However, the signals are too low for sensitive detection. Therefore, we improved the immobilization efficiency by modification of the gold surface with reactive electrophilic groups to increase the surface density of PTOs. The results in Table 3 indicate that the immobilization of PT-functionalized antitags is improved by chemically functionalizing the gold surface.

3.3 Measurements on chemically modified gold surfaces Iodoacetyl and iodosilane functionalized gold surfaces were prepared as described in Section 2.1. Measurements on these surfaces were performed analogous to the measurements described in Section 3.2. The hybridization signals for the iodoacetyl surface are shown in Fig. 7. 

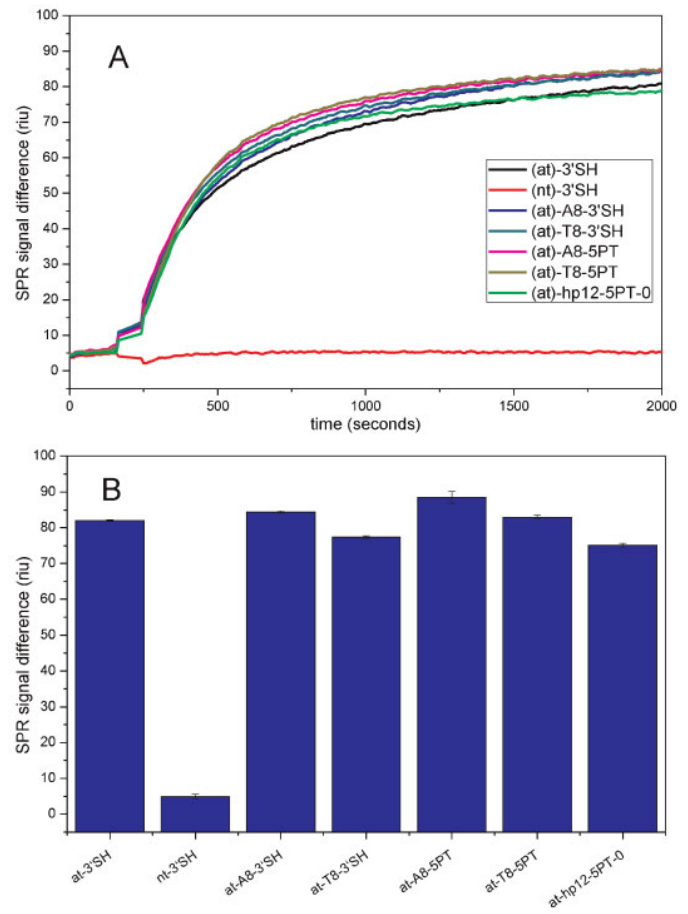

Figure 7 Hybridization kinetics (A) and signal difference in riu (B) from antitags immobilized on an iodoacetyl-functionalized gold surface.

The results show an improvement of the PT modified antitag immobilization in comparison to the SH-modified antitags. The results in Table 3 confirms that this a direct effect of a better antitag immobilization on iodoacetyl functionalized chips in comparison to bare gold surfaces.

Furthermore, there is a general improvement in signal strength in comparison to the results obtained using bare gold surfaces (Fig. 6). This is due to the improved immobilization of antitags summarized in Table 3.

However, PT-modified hairpin structured antitags show a more pronounced improvement in hybridization signal as compared to the improvement in signal strength of linear antitags. These effects are clear when comparing the results in Fig. 6 to those in Fig. 7. However, when looking at the surface coverage values in Table 3 , it can be concluded that although the surface coverage for hairpin structured antitags is improved by functionalization of the gold surface, the actual coverage is still significantly lower as compared to the coverage of linear antitag. Despite this, the signal strength for hp structured antitags is in the same range as the signal for linear antitags. This has to be a direct consequence of a more efficient hybridization due to a better orientation of the antitags on the iodoacetyl functionalized chip surface.

In Fig. 8, the hybridization signals are shown for the iodosilane-functionalized gold surface, the results correlate well with the surface coverage values obtained from the experiments summarized in Table 3. The results show a relative increase in signal strength for PT-modified antitags.
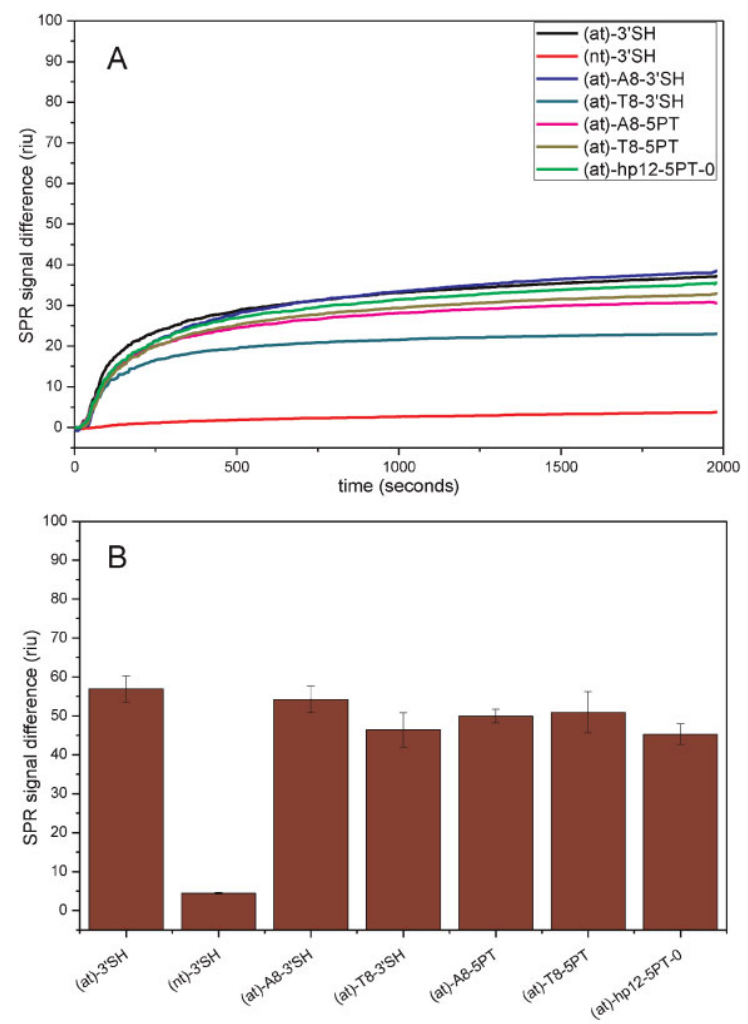

Figure 8 Hybridization kinetics (A) and signal difference in riu (B) from antitags immobilized on an iodosilane-functionalized gold surface.

Moreover, the effect is most pronounced for hairpin structured antitags, as their signal is in the same range although Table 3 clearly shows a lower antitag surface coverage. Comparing the results to the results obtained when hybridizing complementary tags to antitags immobilized on iodoacetylfunctionalized gold surfaces, it can be concluded that this results in lower signal strengths. This can be explained by the lower antitag surface coverage on these surfaces.

3.4 Hybridization kinetics In order to compare the measured kinetic curves, we also performed a mathematical fit based on the concept of "hybridization time constants" $(\tau)$, in analogy to the "denaturation time constant" introduced earlier by van Grinsven et al. [22]. The increase in SPR signal is solely based on hybridization, meaning that a single exponential fit can be used for analyzing the data as shown in Eq. (1),

$$
\operatorname{SPR}(t)=\operatorname{SPR}(t=\infty)-A \exp \left\{-\frac{t}{\tau}\right\}
$$

The hybridization kinetics of (at)-3'SH on three different surfaces was analyzed: bare gold as a reference, gold functionalized with iodoacetyl or with iodosilane. The real time kinetics of the SPR signal are depicted in Fig. 9, where the red line indicates the fit to the data performed with Eq. (1). 


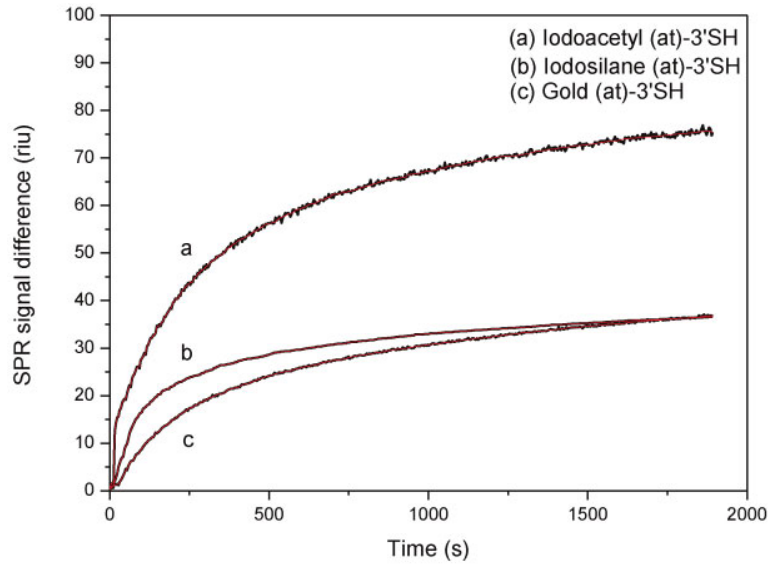

Figure 9 Real time kinetics of the SPR signal for (at)-3'SH on three different surfaces: (a) iodoacetyl-functionalized gold surface, (b) iodosilane-functionalized gold surface, and (c) bare gold surface. The red line indicates the exponential fit on the data points shown in black.

From Fig. 9 it can be obtained that the signal strength is the highest for hybridization with antitags immobilized on an iodoacetyl-functionalized gold surface, with an $A$-value of $89.3 \pm 0.8$ riu.

The respective $A$-values for hybridization with "at" immobilized on an iodosilane-functionalized gold surface and a bare gold surface are $59.2 \pm 1.1$ and $48.7 \pm 0.3$ riu. The $\tau$-value for hybridization of the tag with "at" immobilized on an iodoacetyl-functionalized gold surface is $6.9 \pm 0.1 \mathrm{~min}$. The $\tau$-values for hybridization with "at" immobilized on an iodosilane-functionalized gold surface and a bare gold surface are $4.7 \pm 0.1$ and $8.2 \pm 0.1 \mathrm{~min}$, respectively. From these data it can be concluded that chemical functionalization with iodoacetyl and iodosilane results in faster hybridization (smaller $\tau$ values) and a higher surface coverage of antitags, and therefore, a higher signal strength (higher $A$ value) as compared to results obtained on bare gold. These findings indicate that chemical functionalization leads to a better hybridization efficiency in addition to a higher immobilization efficiency, since a higher antitag surface coverage should normally result in a longer reaction time, and hence, a higher time constant. Chemical modification of the surface of the SPR chips seems to lead to a better orientation of the antitags, and therefore, an improved hybridization efficiency.

The same analysis was conducted for each of the antitags mentioned in Table 1 on all three surfaces. The results obtained by SPR analysis are analyzed by the same exponential fit and are summarized in Table 4. All time constants were found to be less than 10 min being in accordance with values found in literature [34, 35].

These results show that hybridization times decrease and signal strengths increase as a result of chemical modification of the gold surface. However, the increased signal strength is most pronounced when functionalizing gold surfaces with iodoacetyl. These findings hold for all antitags used during the experiment and confirm the results obtained above.

Looking at the data for hybridization of a tag with (nt)3'SH immobilized on a iodoacetyl functionalized gold surface or a bare gold surface, it seems that hybridization occurred very fast (time constants $<3 \mathrm{~min}$ ). However, the signal strength is much lower in comparison to the effect of hybridization to complementary antitags, meaning that nonspecific binding of (nt)-3'SH will result in a small signal. Therefore, it will take less time for the signal to reach a stable value again, which is translated into a lower time constant.

Combining the results summarized in Table 4 with the results obtained in previous paragraphs, it can be concluded that iodoacetyl functionalization of gold chips is the best approach to increase both antitag surface coverage and hybridization efficiency. Furthermore, hybridization times are decreased in comparison to hybridization to antitags on bare gold surfaces. As the time constants are comparable for both iodoacetyl and iodosilane functionalized gold surfaces but the signal strength is better in the first case, iodoacetyl functionalized gold SPR chips should be the surface of choice when developing a biosensor set-up.

4 Conclusions In this paper a lab-on-a-chip system was used for analyzing the effect of using different chemically functionalized gold surfaces on hybridization kinetics of complementary tags to antitags.

The results provided by the reference measurements on bare gold in this article clearly state that PT-functionalized antitags with or without hairpin structures display lower signal strengths in comparison to $\mathrm{SH}$-functionalized linear

Table 4 Fit results obtained from hybridization with different antitags on different surfaces.

\begin{tabular}{|c|c|c|c|c|c|c|}
\hline \multirow[t]{2}{*}{ antitag } & \multicolumn{2}{|l|}{ gold } & \multicolumn{2}{|l|}{ iodoacetyl } & \multicolumn{2}{|l|}{ iodosilane } \\
\hline & $\tau(\min )$ & $A$ (riu) & $\tau(\min )$ & $A$ (riu) & $\tau(\min )$ & $A$ (riu) \\
\hline (at) $-3^{\prime} \mathrm{SH}$ & $8.2 \pm 0.1$ & $48.7 \pm 0.3$ & $6.9 \pm 0.1$ & $89.3 \pm 0.8$ & $4.7 \pm 0.1$ & $59.2 \pm 1.1$ \\
\hline (nt)-3'SH & $1.5 \pm 0.2$ & $8.6 \pm 2.6$ & $2.0 \pm 0.2$ & $14.0 \pm 2.3$ & $24.5 \pm 0.5$ & $4.2 \pm 0.03$ \\
\hline (at)-A8-3'SH & $6.1 \pm 0.1$ & $35.2 \pm 0.2$ & $7.0 \pm 0.1$ & $58.3 \pm 0.3$ & $7.1 \pm 0.1$ & $35.6 \pm 0.3$ \\
\hline (at)-T8-3'SH & $8.7 \pm 0.1$ & $51.9 \pm 0.2$ & $5.5 \pm 0.1$ & $109.8 \pm 0.8$ & $5.6 \pm 0.1$ & $14.6 \pm 0.1$ \\
\hline (at)-A8-5PT & $8.2 \pm 0.1$ & $43.2 \pm 0.2$ & $5.1 \pm 0.1$ & $116.3 \pm 1.0$ & $4.5 \pm 0.1$ & $46.0 \pm 0.7$ \\
\hline (at)-T8-5PT & $7.3 \pm 0.1$ & $27.6 \pm 0.2$ & $4.8 \pm 0.04$ & $127.1 \pm 0.9$ & $4.9 \pm 0.1$ & $48.2 \pm 0.6$ \\
\hline (at)-hp12-5PT-0 & $7.4 \pm 0.1$ & $24.3 \pm 0.2$ & $4.7 \pm 0.03$ & $119.4 \pm 0.8$ & $5.3 \pm 0.1$ & $48.0 \pm 0.6$ \\
\hline
\end{tabular}


antitags. This can be explained by the fact that SHfunctionalized antitags show a higher antitag surface coverage as compared to PT-functionalized antitags. In order to improve the surface coverage of PT-functionalized antitags on the SPR chip and to improve the orientation of hairpin structured PT-functionalized antitags, two different chemical functionalization methods were analyzed.

The experiments on chemically functionalized gold surfaces show that hybridization to antitags immobilized on these surfaces occurs faster in comparison to results obtained in reference measurements. However, iodoacetyl functionalization of gold surfaces results in an increased SPR signal. This can be attributed to the fact that iodoacetyl functionalization results in a higher surface coverage of antitags on the surface. Moreover, the increase in signal strength and decrease in hybridization time is more pronounced when using PTOs with hairpin structures as an improvement of the orientation of these antitags leads to a more efficient hybridization in addition to a higher antitag surface coverage.

For the implementation into a biosensor for the detection of single nucleotide polymorphisms (SNPs), it is possible to immobilize PT-functionalized, hairpin-structured antitags onto an iodoacetyl-functionalized gold surface. The PT functionalization will make the antitags more resistant toward enzymatic degradation by DNA endonucleases. In addition, the hairpin structures ensure a higher selectivity of the antitag for full match tags in the sample under analysis.

Acknowledgements This work was financially supported by the BMBF (German Ministry of Education and Research, grant 03WKBH2: "SPR-Biochips").

\section{References}

[1] J. Homola, Chem. Rev. 108, 462-493 (2008).

[2] J. Kafka, O. Pänke, B. Abendroth, and F. Lisdat, Electrochim. Acta 53, 7467-7474 (2008).

[3] O. Pänke, A. Kirbs, and F. Lisdat, Biosens. Bioelectron. 22, 2656-2662 (2007).

[4] E. L. S. Wong and J. J. Gooding, Anal. Chem. 78, 2138-2144 (2006).

[5] A. J. Thiel, A. G. Frutos, C. E. Jordan, R. M. Corn, and L. M. Smith, Anal. Chem. 69, 4948-4956 (1997).

[6] F. Caruso, E. Rodda, and D. N. Furlong, Anal. Chem. 69, 2043-2049 (1997).

[7] T. M. Herne, M. J. Tarlov, and J. Am, Chem. Soc. 119, 89168920 (1997).

[8] C. Bamdad, Biophys. J. 75, 1997-2003 (1998).

[9] C. A. Stein, C. Subasinghe, K. Shinozuka, and J. S. Cohen, Nucleic Acids Res. 16, 3209-3221 (1988).

[10] R. P. Iyer, W. Eagan, J. B. Reagan, and S. L. Baucage, J. Am. Chem. Soc. 112, 1253-1254 (1990).

[11] D. J. Duggan, M. Bittner, Y. Chen, P. Meltzer, and J. M. Trent, Nature Genet. 21 (Suppl), 10-14 (1999).

[12] X. Zhao, S. Nampalli, A. J. Serino, and S. Kumar, Nucleic Acids Res. 29, 955-959 (2001).
[13] R. Bordoni, C. Consolandi, B. Castiglioni, E. Busti, L. R. Bernardi, C. Battaglia, and G. De Bellis, Nucleic Acids Res. 8, e34 (2002).

[14] A. Kumar, S. Phadtare, R. Pasricha, P. Guga, K. N. Ganesh, and M. Sastry, Curr. Sci. India 1, (2003).

[15] L. Jiang, H. Zhang, J. Zhuang, and B. Yang, Adv. Mater. 17, 2066-2070 (2005).

[16] B. Yang, L. Jiang, J. Zhuang, and Y. Liu, Chin. J. Chem. 23, 1143-1145 (2005).

[17] I. Lubitz and A. Kotlyar, Bioconjugate Chem. 22, 2043-2047 (2011).

[18] J. Ghabboun, M. Sowwan, H. Cohen, T. Molotsky, N. Borovok, B. Dwir, E. Kapon, A. Kotlyar, and D. Porath, Appl. Phys. Lett. 91, 173101 (2007).

[19] H. Ceretti, B. Ponce, S. A. Ramírez, and J. M. Montserrat, Electroanalysis 22, 147-150 (2010).

[20] J. H. Lee, D. P. Wernette Dr, M. V. Yigit, J. Liu, Z. Wang, and Y. Lu, Angew. Chem., Int. Ed. 46, 9006-9010 (2007).

[21] P. Nilsson, B. Persson, M. Uhlén, and P. Nygren, Anal. Biochem. 224, 400-408 (1995).

[22] B. van Grinsven, N. van den Bon, L. Grieten, M. Murib, S. D. Janssens, K. Haenen, E. Schneider, S. Ingenbrandt, M. J. Schöning, V. Vermeeren, M. Ameloot, L. Michiels, R. Thoelen, W. De Ceuninck, and P. Wagner, Lab Chip 11, 1656-1663 (2011).

[23] F. Sonntag, S. Schmieder, N. Danz, M. Mertig, N. Schilling, U. Klotzbach, and E. Beyer, Proc. SPIE Bioeng. Bioinspired Syst. IV, 7365, 7365 0Q-1-73650Q-9 (2009).

[24] M. Mertig, A. Kick, M. Bönsch, B. Katzschener, J. Voigt, F. Sonntag, N. Schilling, U. Klotzbach, N. Danz, S. Begemann, A. Herr, and M. Jung, Sens. IEEE 392-395, 2009.

[25] A. Kick, M. Bönsch, B. Katzschner, J. Voigt, A. Herr, W. Brabetz, M. Jung, F. Sonntag, U. Klotzbach, N. Danz, S. Howitz, and M. Mertig, Biosens. Bioelectron. 26, 1543 1547 (2010).

[26] F. Diehl, S. Grahlmann, M. Beier, and J. D. Hoheisel, Nucleic Acids Res. 29, (2001).

[27] B. Tinland, A. Pluen, J. Sturm, and G. Weill, Macromolecules 30, 5763-5765 1997.

[28] V. Chan, D. J. Graves, and S. E. McKenzie, Biophys. J. 69, 2243-2255 (1995).

[29] B. van Grinsven, N. Vanden Bon, H. Strauven, L. Grieten, M. Murib, K. L. Jiménez-Monroy, S. D. Janssens, K. Haenen, M. J. Schöning, V. Vermeeren, M. Ameloot, L. Michiels, R. Thoelen, W. De Ceuninck, and P. Wagner, ACS Nano 6, 2712-2721 (2012).

[30] V. Vermeeren, S. Wenmackers, M. Daenen, K. Haenen, O. Williams, M. Ameloot, M. Vande Ven, P. Wagner, and L. Michiels, Langmuir 24, 9125-9134 (2008).

[31] P. V. Riccelli, F. Merante, K. T. Leung, S. Bortolin, R. L. Zastawny, R. Janeczko, and A. S. Benight, Nucleic Acids Res. 29, 996-1004 (2001).

[32] H. Du, M. D. Disney, L. Miller, and T. D. Krauss, J. Am. Chem. Soc. 125, 4012-4013 (2003).

[33] N. E. Broude, Trends Biotechnol. 20, 249-256 (2002).

[34] A. W. Peterson, R. J. Heaton, and R. M. Georgiadis, Nucleic Acid Res. 29, 5163-5168 (2001).

[35] F. F. Bier, F. Kleinjung, and F. W. Scheller, Sens. Actuators, B 38-39, 78-82 (1997). 